\title{
Structure-Dependent 4-Tert-Butyl Pyridine-Induced Band Bending at $\mathrm{TiO}_{2}$ Surfaces
}

\author{
Mats Göthelid, ${ }^{1}$ Shun Yu, ${ }^{1}$ Sareh Ahmadi, ${ }^{1}$ Chenghua Sun,, ${ }^{1,2}$ and Marcelo Zuleta ${ }^{3}$ \\ ${ }^{1}$ Materialfysik, ICT, KTH, Electrum 229, 16440 Stockholm, Sweden \\ ${ }^{2}$ The University of Queensland, ARC Centre of Excellence for Functional Nanomaterials and Centre for Computational Molecular \\ Science, Australia Institute for Bioengineering and Nanotechnology, The University of Queensland, Qld 4072, Australia \\ ${ }^{3}$ Physical \& Analytical Chemistry Department, Uppsala University, Lägerhyddsvägen 1, 75237 Uppsala, Sweden \\ Correspondence should be addressed to Mats Göthelid, gothelid@kth.se
}

Received 31 May 2010; Accepted 17 August 2010

Academic Editor: J. Anthony Byrne

Copyright (๑) 2011 Mats Göthelid et al. This is an open access article distributed under the Creative Commons Attribution License, which permits unrestricted use, distribution, and reproduction in any medium, provided the original work is properly cited.

The role of 4-tert butyl pyridine (4TBP) adsorption on $\mathrm{TiO}_{2}$ surface band bending has been studied using photoelectron spectroscopy. Surface oxygen vacancies pin the Fermi level near the conduction band edge on rutile (110). 4TBP preferentially adsorbs in those vacancies and shift the Fermi level to lower binding energy in the band gap. This is done by transferring vacancy excess charge into the empty $\pi^{*}$ orbital in the pyridine ring. The anatase (100) surface contains much less oxygen vacancies although the surface is much rougher than the rutile (110). 4TBP adsorption does not have any significant effect on the surface band bending. Thus the positive role associated with 4TBP addition to solar cell electrolytes is suggested to protection against adsorption of other electrolyte components such as Li and I.

\section{Introduction}

Dye sensitized solar cells (DSSCs) are very complex with a working electrode made of a $\mathrm{TiO}_{2}$ nanostructured network decorated by light harvesting dye molecules contacted to a counter electrode through an electrolyte [1]. It was discovered many years ago that addition of 4-terbutyl pyridine (4TBP) to an $\left(\mathrm{I}_{3}{ }^{-} / \mathrm{I}^{-}\right)$electrolyte increases the fill factor and the open circuit voltage $\left(V_{o c}\right)$ from 0.38 to $0.72 \mathrm{~V}$, while the short circuit current is not influenced [2]. Several explanations have been put forward, including 4TBPinduced band bending towards lower binding energies [3], reduction of the amount of adsorbed protons, $\mathrm{Li}^{+}$and/or other cations present in the electrolyte $[4,5]$, decreasing the electron recombination from $\mathrm{TiO}_{2}$ to tri-iodide in the electrolyte by blocking the tri-iodide from reaching the surface [5] or removing adsorbed iodine [6].

Surface or interface defects are known to alter the electrical properties of semiconductor contacts [7] and this is the case also for DSSC's. Oxygen vacancies are commonly observed on oxides and they play a significant role on the surface properties $[8,9]$. Naturally the density and character of those defects determine the Fermi level position at the interface. The removal of oxygen from $\mathrm{TiO}_{2}(110)$ leave charged vacancies with $\mathrm{Ti}^{3+}$ ions. This process generates surface states located within the band gap, $\sim 1 \mathrm{eV}$ below the Fermi level, that controls the Fermi level position [8, $10,11]$. Measurements on nanocrystalline $\mathrm{TiO}_{2}$ indicated that 4 TBP reduces the density of defects and the charge carrier recombination [12], through bonding to specific sites. Whether 4TBP prefers to adsorb at those "specific sites" or not, and whether this is the essential reason for the band shift have not been clarified. This is not an easy task since solar cells contain nanocrystalline $\mathrm{TiO}_{2}$ which has the potential to hold a multitude of various structures and defects; thereby it is very difficult, not to say impossible, to make a unique identification of the essential contribution of each and every one of those defects to the observed shift. Although single crystal substrates are far from realistic in a solar cell they allow for preparation of surfaces with reasonably well controlled amounts and types of defects. In a recent paper we showed that $4 \mathrm{TBP}$ prefers to bind directly to oxygen vacancies on rutile $\mathrm{TiO}_{2}(110)$ giving a $0.2 \mathrm{eV}$ upward shift of the band edges through a combination of photoelectron 


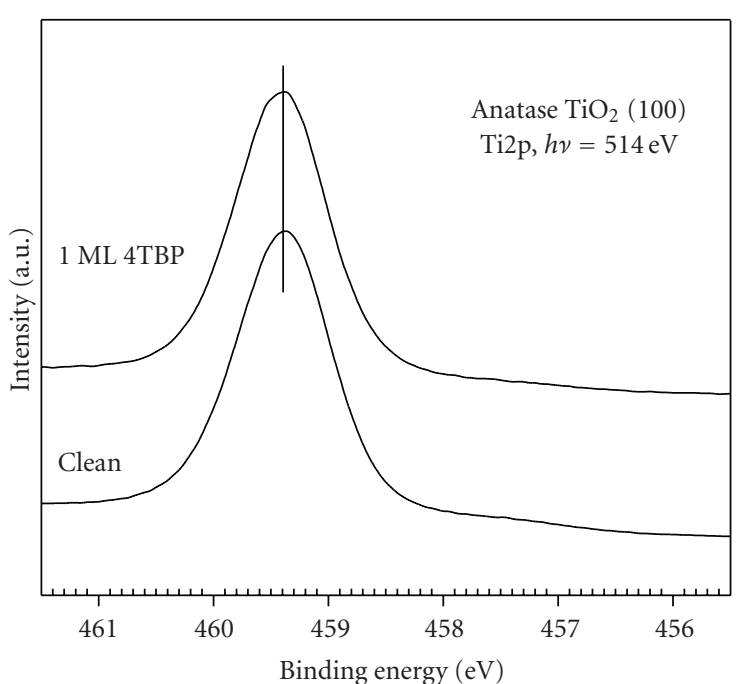

(a)

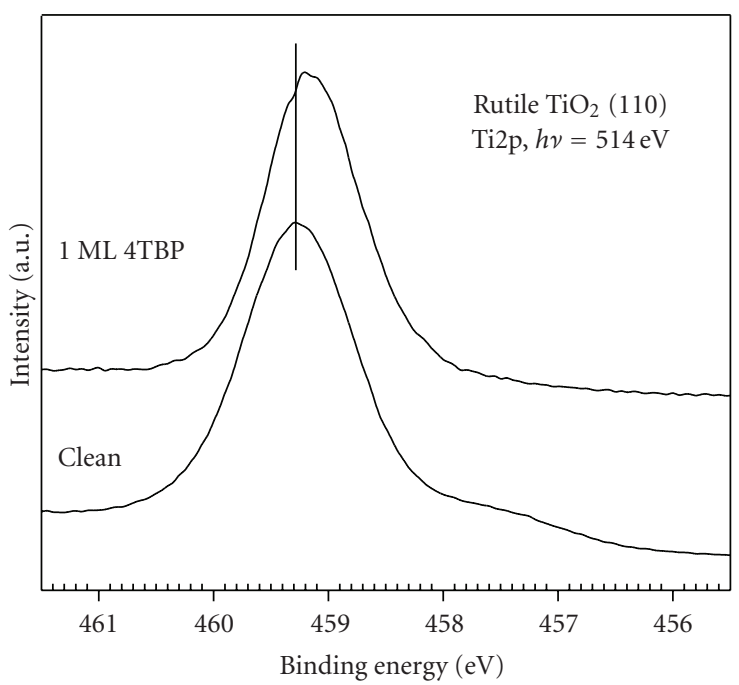

(b)

FIgure 1: Ti2p spectra from clean and 4TP-covered $\mathrm{TiO}_{2}(100)$ and $\mathrm{TiO}_{2}(110)$ surfaces. The photon energy was $514 \mathrm{eV}$.

spectroscopy (PES) and density functional theory (DFT) [13]. In this paper, we extend this study to compare with anatase $\mathrm{TiO}_{2}(100)$. Contrary to the rutile surface we do not observe oxygen vacancies and the band edges do not shift upon 4TBP adsorption. Based on the present result one can suggest that the major role of 4TBP in DSSC electrolyte is to protect the surface from adsorption of ionic components in the electrolyte. However, more work is needed to clarify this in detail since nanostructured $\mathrm{TiO}_{2}$ contains many crystal planes and adsorption sites.

\section{Experimental Methods}

Experiments were done at beamline I511-1, MAX-lab, Lund, Sweden. This is an undulator-based beamline giving horizontally polarized light between 100 and $1200 \mathrm{eV}$ selected by a modified Zeiss SX-700 monochromator. Photoelectron spectra were recorded using a Scienta R4000 spectrometer which is rotatable around the axis of the photon beam in the analysis chamber. The preparation chamber holds a sputter gun, sample annealing, and low energy electron diffraction (LEED) optics. The base pressure was lower than $1 \times 10^{-10}$ mbar. A load lock chamber is connected to the preparation chamber for fast sample entry and molecule deposition.

The rutile $\mathrm{TiO}_{2}(110)$ crystal was aligned to within $0.2^{\circ}$ from the (110) plane and purchased from Surface Preparation Laboratory, the Netherlands. Thermal treatment above $1000 \mathrm{~K}$ for two hours in UHV generates bulk oxygen vacancies, changing the sample color from transparent to blue and increasing the conductivity. Before each deposition, the surface was cleaned by several rounds of $\mathrm{Ar}^{+}$sputtering followed by annealing in UHV, which gave a sharp $(1 \times 1)$ LEED pattern. The anatase sample was $10 \times 3 \times 1 \mathrm{~mm}^{3}$ aligned to $\approx 0.1^{\circ}$ from the (100) plane purchased from Surface Preparation Laboratory. The sample was cleaned by repeated cycles of $\mathrm{Ar}^{+}$-sputtering and annealing at $550^{\circ} \mathrm{C}$, until a sharp LEED pattern was obtained. The sputtering was conducted varying the sputter energy from low energy (50$400 \mathrm{eV}$ ) to higher energy up to $1000 \mathrm{eV}$. The argon pressure, $\mathrm{P}_{\mathrm{Ar}^{+}}$, was $2.0 \cdot 10^{-7}$ mbar. The annealing was performed during $45 \mathrm{~min}$.

4TBP (Sigma-Aldrich, 99\% purity) was dosed, after a few pumping cycles, at room temperature through a precision leak valve on the load lock. The base pressure in the load lock was $6 \times 10^{-10} \mathrm{mbar}$. During deposition the substrate was held at room temperature. Formation of the saturated single monolayer is reached when spectra do change, neither from molecule nor from substrate after further exposure. Photoelectron spectra of core level and valence band were collected after each deposition. To avoid beam damage to the organic layers, the sample was moved $(0.1 \mu \mathrm{m} / \mathrm{min})$ during $\mathrm{X}$-ray exposure. The energy scale was calibrated to the Fermi level, recorded from a tantalum foil in electrical contact with the sample.

\section{Results and Discussion}

A set of Ti2 $p_{3 / 2}$ spectra is plotted in Figure 1, the left hand side from anatase $\mathrm{TiO}_{2}(100)$ and the right hand side from rutile $\mathrm{TiO}_{2}(110)$. The lower spectra are from the clean substrate and spectra above after deposition of one 4TBP monolayer (ML). On the clean anatase surface the bulk peak lies at $459.4 \mathrm{eV}$, while on the rutile surface it is at $459.3 \mathrm{eV}$. In the spectrum from the rutile surface there is an additional weak hump around $457.5 \mathrm{eV}$ due to $\mathrm{Ti}^{3+}$, either at the surface or in the bulk. The surface related $\mathrm{Ti}^{3+}$ ions are located in the oxygen vacancies, generated from removal of bridging oxygen. This peak is hardly visible on anatase, suggesting a much lower density of oxygen vacancies. On a well-prepared rutile (110) surface the density of oxygen vacancies is around $8 \%$ [14]. Adsorption of one ML of 4TBP on anatase (100) 


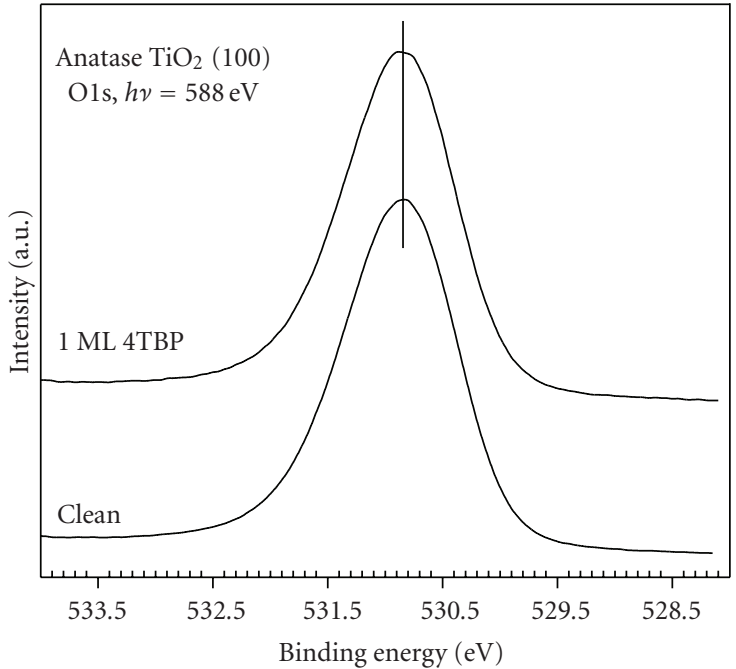

(a)

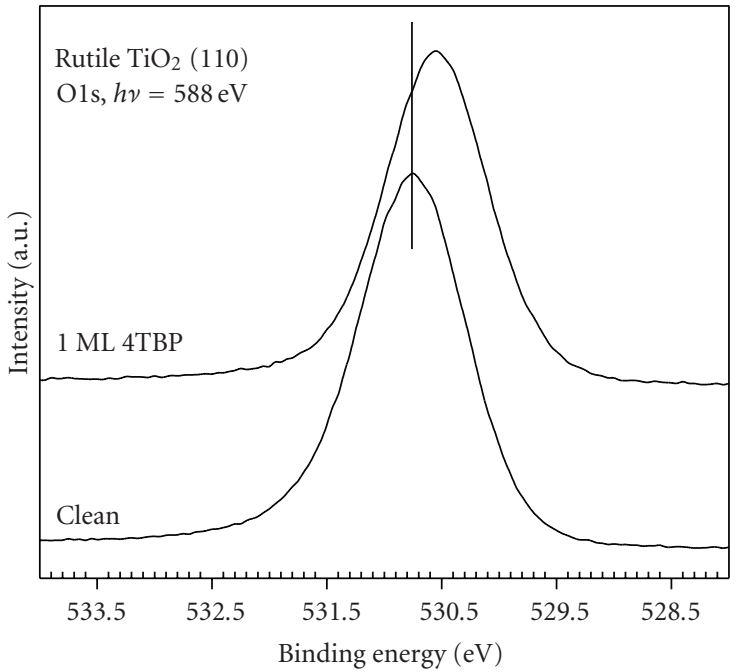

(b)

FIgURE 2: O1s spectra from clean and $4 \mathrm{TP}$ covered $\mathrm{TiO}_{2}(100)$ and $\mathrm{TiO}_{2}(110)$ surfaces. The photon energy was $588 \mathrm{eV}$.

does not induce any shape changes of the spectral line; moreover the binding energy is unaffected and no new shifted peaks appear. On the contrary on rutile (110) the $\mathrm{Ti}^{3+}$ related component is strongly reduced, which even happens at low coverage [13]. This result clearly indicates that the $\mathrm{O}$-vacancy is a preferred bond site for 4TBP. In addition, the main $\mathrm{Ti}^{4+}$ peak shifts $0.2 \mathrm{eV}$ to lower binding energy. Such shifts indicate adsorption-induced band bending, in line with previous work [3-6].

Figure 2 shows O1s spectra from the same surfaces and preparations as in Figure 1. Spectra from the pristine surfaces, bottom spectra, contain only one component at $530.85 \mathrm{eV}$. Upon adsorption of $1 \mathrm{ML}$ of 4TBP the line shapes are essentially unaffected. The binding energy on anatase does not change while $\mathrm{O} 1 \mathrm{~s}$ from rutile shifts to lower binding energy by $0.25 \mathrm{eV}$. This result agrees with Ti2p, and 4TBP induces surface band bending on rutile but not on anatase. It was previously observed that O1s shifts back to lower binding energy after healing the oxygen vacancies with oxygen [11]. Our observation of a shift to lower binding energy and the removal of $\mathrm{Ti}^{3+}$ from Ti2p spectra support that finding and suggest that $4 \mathrm{TBP}$ reduces the surface charge on rutile $\mathrm{TiO}_{2}$.

$\mathrm{C} 1 \mathrm{~s}$ spectra from three different coverages are shown in Figure 3; 0.02 ML, 0.2 ML, and $1 \mathrm{ML}$ from both substrates. The coverage was determined from setting the intensity at saturation to $1 \mathrm{ML}$. Other spectra were normalized to this intensity. There may be errors related to this treatment, in particular for the lowest coverage, but the exact coverage is not of central importance here. The spectra from the lowest coverage are broader than that at monolayer coverage, but no shifted components are resolved. What is clear though is a shift to higher binding energy with increasing coverage. The monolayer binding energies are $286.2 \mathrm{eV}$ on anatase and $285.9 \mathrm{eV}$ on rutile, while the $0.2 \mathrm{ML}$ binding energies are $285.6 \mathrm{eV}$ on anatase and $285.3 \mathrm{eV}$ on rutile and finally at the lowest coverage the binding energies are $285.3 \mathrm{eV}$ on anatase and 285.05 on rutile. Thus there is a general trend that binding energies are lower on rutile than on anatase by $0.3 \mathrm{eV}$. Therefore, it can be concluded that the coveragedependent shifts to higher binding energy are same for the two surfaces.

It is known from previous studies that $4 \mathrm{TBP}$ adsorbs in an upright geometry with nitrogen coordinating to incompletely coordinated titanium: as suggested from Raman spectroscopy, IR spectroscopy and XPS on nanocrystalline anatase [6] and density functional theory for 4TBP on $\mathrm{TiO}_{2}(110)$ [15]. Furthermore, our previous DFT optimizations showed that 4TBP prefers standing up both on fivefold coordinated $\mathrm{Ti}\left(\mathrm{Ti}^{5 f}\right)$ and oxygen vacancies, even if other geometries are set as the starting geometry [13]. The bonding has two main contributions; a $\sigma$-type Ti-N contribution and a Ti3d- $\pi^{*}$ contribution.

The band bending to lower binding energy implies a charge transfer from the oxygen vacancies into the 4TBP empty $\pi^{*}$ orbitals on the carbon ring. Such charge transfer ceases when the vacancies are filled, which is seen in the saturation of the Ti2p and O1s core level shifts, that is, the band bending, while the $\mathrm{C} 1 \mathrm{~s}$ core level continues to shift with the adsorption of 4TBP. Using the core level binding energy as a simple measure of the local electron density, it is found that higher surface density of 4TBP reduces the average charge on the molecules. This is not surprising and can be understood as due to dipole repulsion, which is distance dependent and charge dependent. The dipole repulsion thus redistributes the charge within the molecular layer and reducing the charge on each molecule allowing a higher density. Our observation thus implies that the $\sigma$-type Ti-N bond dominates the Ti3 $\mathrm{d}-\pi^{*}$ bond in determining the bond site and bond strength.

The coverage-dependent shift is similar on anatase despite the lack of band bending and oxygen vacancies. This would suggest that the molecule-molecule repulsion is similar within the 4TBP layer. It also indicates that the molecular dipoles are comparable and that the average 


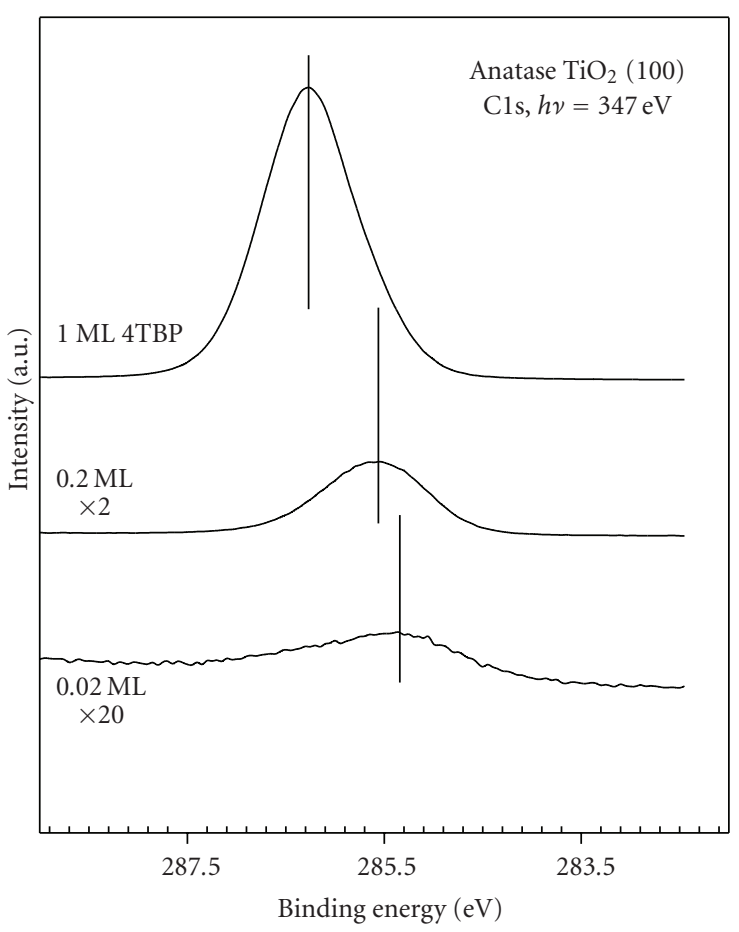

(a)

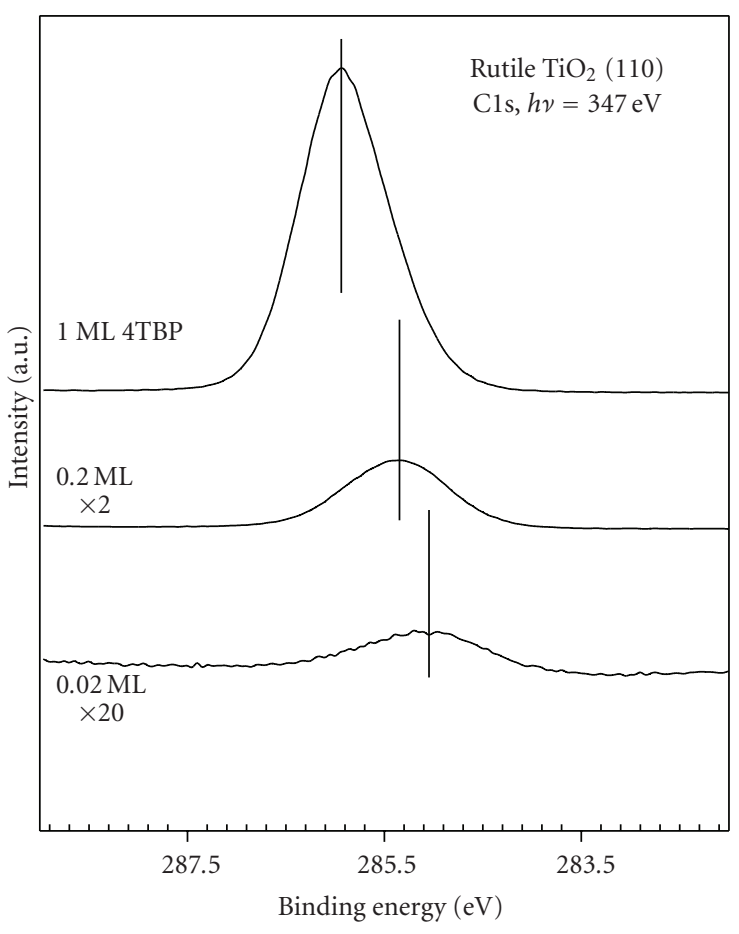

(b)

Figure 3: C1s spectra from 4TBP at different coverages. Incident photon energy $347 \mathrm{eV}$.

charge transferred is similar. On the other hand, there is a difference in binding energy, $0.3 \mathrm{eV}$ higher on anatase than on rutile. This could indicate a larger charge transfer on rutile than on anatase, presumably due to the oxygen vacancies. However, the binding energy of a core level is a complex function of charge density, initial state effects, and flexibility in the system upon ionization, final state effects. The screening of the ionic molecule will be influenced by the substrate, to the distance and availability and mobility of electrons. On a semiconductor it will also be influenced by the Fermi level position and surface band bending. Taking the core level to valence band edge as a measure of the core level binding energy we can conclude that the $\mathrm{C} 1 \mathrm{~s}$ binding energy on rutile shifts more than that on anatase, thus supporting a larger charge transfer due to the oxygen vacancies. It is also clear that there is a coverage-dependent variation in the charge density on the carbon ring and thus charge transfer does happen on anatase, just not large enough to induce band bending as observed on rutile.

Spectra from the top of the valence band (VB) from clean and 4TBP covered surfaces are shown in Figure 4. This region includes the valence band edge and the vacancy-induced state in the band gap. Note that the intensity scales are the same in spectra from the same surface but different in spectra from the two surfaces. This is to enhance line profile. The gap state is located at approximately $0.9 \mathrm{eV}$ below Fermi level on rutile and is attributed to nonbonding Ti3d electrons at oxygen vacancies. Other origins have been discussed, but the oxygen vacancy is by far the largest contributor on $\mathrm{TiO}_{2}(110)$ $[9,16,17]$.
Adsorption of $1 \mathrm{ML} 4 \mathrm{TBP}$ on rutile shifts of the whole VB towards the vacuum level by $\sim 0.2 \mathrm{eV}$, as seen at the band edge just above $3.1 \mathrm{eV}$ binding energy. The edge is not sharp and the exact binding energy position is not easily extracted, especially after adsorption. Instead we use the shift of a stronger peak in the valence band (not shown). Adsorption of 4TBP also reduces the surface state intensity. This reduction is much stronger than the intensity reduction of the rest of the valence band (not shown). However, the gap state is not completely removed and the remains may be assigned to other kinds of defects such as subsurface vacancies and interstitials that are not within reach for the 4TBP [17].

On anatase the intensity of the state is lower than on rutile and it does not change upon $4 \mathrm{TBP}$ adsorption, see Figure 4(a). This suggests that oxygen vacancies are not present on this surface, or that if they are they do not play a significant role in the adsorption process. The small intensity thus stems from subsurface vacancies, interstitials, or impurities. The anatase sample was cut from a natural crystal and may contain small amounts of impurities. The band edge at $3.25 \mathrm{eV}$ stays firm, in agreement with the absence of core level shifts in Ti2p and O1s. The peak at $1 \mathrm{eV}$ also retains its binding energy. One striking observation is the Fermi edge, the surface is metallic. This is not visible; the intensity scale is adapted for the whole valence band spectrum. However, when scaled differently it is obvious. A possible reason for this metallicity is subsurface impurities, which varies between samples from different sources and preparation methods. 


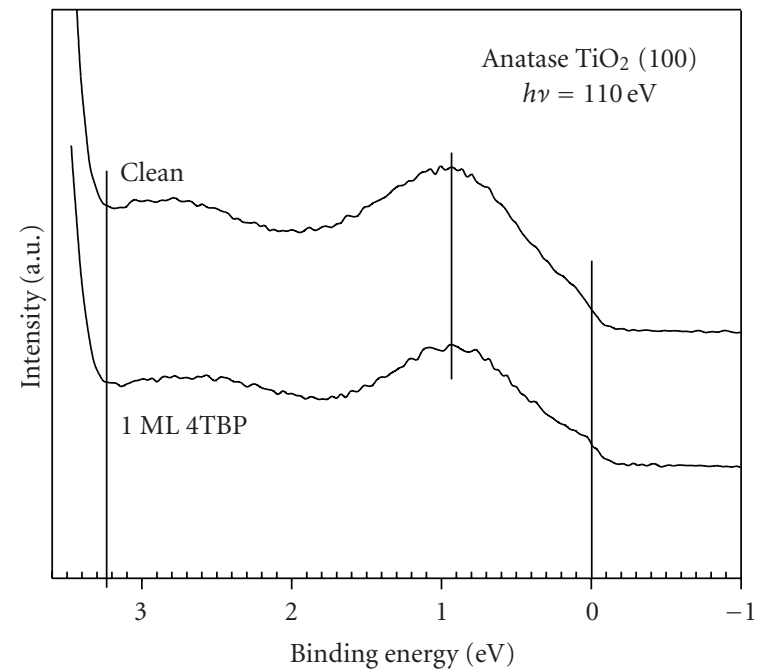

(a)

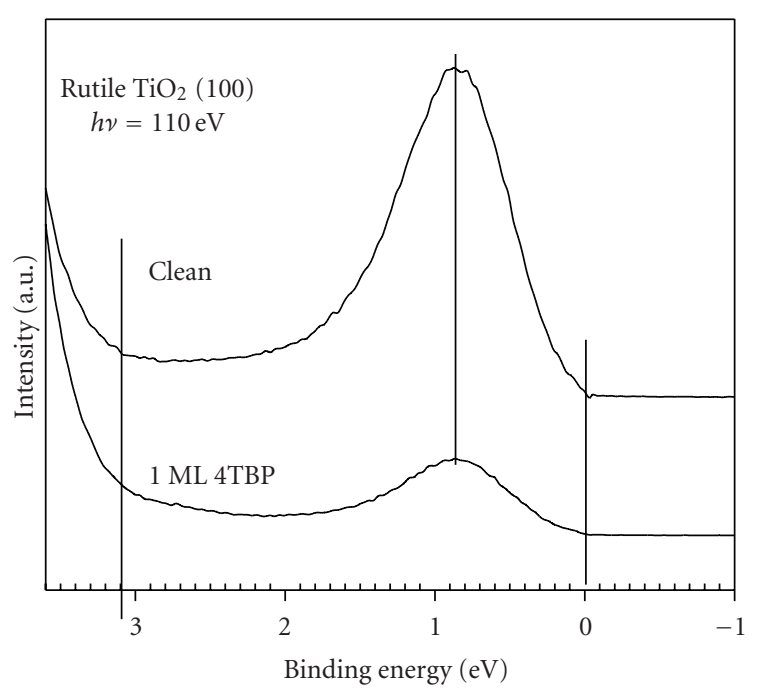

(b)

Figure 4: Spectra from the top of the valence band and the Fermi level region from $4 \mathrm{TBP} / \mathrm{TiO}_{2}$ interfaces compared with that of clean samples.

\section{Summary and Conclusions}

The appearance of oxygen vacancies on rutile $\mathrm{TiO}_{2}(110)$ plays a key role in determining the Fermi level position. The removal of oxygen leaves a charged vacancy with two unpaired electrons in the $3 \mathrm{~d}$ orbitals $[18,19]$. 4TBP adsorbs preferentially in those vacancies and moves the surplus charge from the surface into the $\pi^{*}$ orbitals on the 4TBP carbon ring. Removal of the additional surface electrons shifts the Fermi level $0.2 \mathrm{eV}$ towards lower binding energy. Following adsorption on five coordinated surface $\mathrm{Ti}$, does not further change the band bending, but the dipole repulsion between $4 \mathrm{TBP}$ redistributes the charge within the molecular layer. This is seen through a coverage-dependent C1s core level shift.

Our results point to a low density of oxygen vacancies on anatase $\mathrm{TiO}_{2}(100)$; the $\mathrm{Ti}^{3+}$ state in the $\mathrm{Ti} 2 \mathrm{p}$ core level and the state in the band gap are much smaller than on rutile, and they do not change upon 4TBP adsorption. Furthermore, the Fermi level position with respect to the band edges is insensitive to 4TBP adsorption. The coverage dependent molecular dipole repulsion is observed also on this surface. From the above discussion the increased output voltage from cells with 4TBP added to the electrolyte [2] cannot be explained by a surface band bending due to oxygen vacancies in the case of anatase. Its positive role as additive in DSSC is suggested to protect the surface from adsorption of ionic components on the electrolyte. However, the solar cell material, nanostructured $\mathrm{TiO}_{2}$, is not made from (100) oriented crystallites and our results do not necessarily represent a realistic situation. More work, both experimental and theoretical, is indeed needed before a final conclusion can be made.

\section{Acknowledgments}

The authors acknowledge Dr. Tomas Edvinsson, Dr. Gerrit Boschloo, Dr. Shanghua Li, and Dr. Jian Qin for helpful discussions. Great thanks go to the kind staff at Max-lab. The Swedish Energy Agency (STEM), the Swedish Research Council (VR), the Göran Gustafsson, Carl Trygger Foundations, and Queensland government are kindly acknowledged for financial support.

\section{References}

[1] B. O’Regan and M. Grätzel, "A low-cost, high-efficiency solar cell based on dye-sensitized colloidal $\mathrm{TiO}_{2}$ films," Nature, vol. 353, no. 6346, pp. 737-740, 1991.

[2] M. K. Nazeeruddin, A. Kay, I. Rodicio et al., "Conversion of light to electricity by cis-X2bis (2,2' -bipyridyl-4,4';dicarboxylate)ruthenium(II) charge-transfer sensitizers ( $\mathrm{X}=$ $\mathrm{Cl}^{-}, \mathrm{Br}^{-}, \mathrm{I}^{-}, \mathrm{CN}^{-}$, and $\mathrm{SCN}^{-}$) on nanocrystalline $\mathrm{TiO}_{2}$ electrodes," Journal of the American Chemical Society, vol. 115, no. 14, pp. 6382-6390, 1993.

[3] G. Schlichthörl, S. Y. Huang, J. Sprague, and A. J. Frank, "Band edge movement and recombination kinetics in dyesensitized nanocrystalline $\mathrm{TiO}_{2}$ solar cells: a study by intensity modulated photovoltage spectroscopy," Journal of Physical Chemistry B, vol. 101, no. 41, pp. 8141-8155, 1997.

[4] S. Nakade, T. Kanzaki, W. Kubo, T. Kitamura, Y. Wada, and S. Yanagida, "Role of electrolytes on charge recombination in dye-sensitized $\mathrm{TiO}_{2}$ solar cell (1): the case of solar cells using the $\mathrm{I}^{-} / \mathrm{I}_{3}^{-}$redox couple," Journal of Physical Chemistry B, vol. 109, no. 8, pp. 3480-3487, 2005.

[5] G. Boschloo, L. Häggman, and A. Hagfeldt, "Quantification of the effect of 4-tert-butylpyridine addition to $\mathrm{I}^{-} / \mathrm{I}_{3}^{-}$redox electrolytes in dye-sensitized nanostructured $\mathrm{TiO}_{2}$ solar cells," Journal of Physical Chemistry B, vol. 110, no. 26, pp. 1314413150, 2006. 
[6] C. Shi, S. Dai, K. Wang, X. Pan, F. Kong, and L. Hu, "The adsorption of 4-tert-butylpyridine on the nanocrystalline $\mathrm{TiO}_{2}$ and Raman spectra of dye-sensitized solar cells in situ," Vibrational Spectroscopy, vol. 39, no. 1, pp. 99-105, 2005.

[7] W. Mönch, Semiconductor Surface and Interfaces, Springer, Berlin, Germany.

[8] A. L. Linsebigler, G. Lu, and J. T. Yates Jr., "Photocatalysis on $\mathrm{TiO}_{2}$ surfaces: principles, mechanisms, and selected results," Chemical Reviews, vol. 95, no. 3, pp. 735-758, 1995.

[9] U. Diebold, "The surface science of titanium dioxide," Surface Science Reports, vol. 48, no. 5-8, pp. 53-229, 2003.

[10] E. L. D. Hebenstreit, W. Hebenstreit, H. Geisler et al., "Sulfur on $\mathrm{TiO}_{2}(110)$ studied with resonant photoemission," Physical Review B, vol. 64, no. 11, Article ID 115418, pp. 1-11, 2001.

[11] K. E. Smith, J. L. MacKay, and V. E. Henrich, "Interaction of $\mathrm{SO}_{2}$ with nearly perfect and defect $\mathrm{TiO}_{2}(110)$ surfaces," Physical Review B, vol. 35, no. 11, pp. 5822-5829, 1987.

[12] M. Dürr, A. Yasuda, and G. Nelles, "On the origin of increased open circuit voltage of dye-sensitized solar cells using 4-ferfbutyl pyridine as additive to the electrolyte," Applied Physics Letters, vol. 89, no. 6, Article ID 061110, 2006.

[13] S. Yu, S. Ahmadi, P. Palmgren, F. Hennies, M. Zuleta, and M. Göthelid, "4-tert-butyl pyridine bond site and band bending on $\mathrm{TiO}_{2}(110)$," Journal of Physical Chemistry C, vol. 114, pp. 2315-2320, 2009.

[14] P. Palmgren, B. R. Priya, N. P. P. Niraj, and M. Göthelid, "Bonding of metal-free phthalocyanine to $\mathrm{TiO}_{2}(110)$ single crystal," Solar Energy Materials and Solar Cells, vol. 90, no. 20, pp. 3602-3613, 2006.

[15] B.-T. Xiong, B.-X. Zhou, Z.-Y. Zhu et al., "Adsorption of 4-tert-butylpyridine on $\mathrm{TiO}_{2}$ surface in dye-sensitized solar cells," Chinese Journal of Chemistry, vol. 26, no. 1, pp. 70-76, 2008.

[16] C. M. Yim, C. L. Pang, and G. Thornton, "Oxygen vacancy origin of the surface band-gap state of $\mathrm{TiO}_{2}(110)$," Physical Review Letters, vol. 104, no. 3, Article ID 036806, 2010.

[17] S. Wendt, P. T. Sprunger, E. Lira et al., "The role of interstitial sites in the Ti3d defect state in the band gap of titania," Science, vol. 320, no. 5884, pp. 1755-1759, 2008.

[18] T. Bredow and G. Pacchioni, "Electronic structure of an isolated oxygen vacancy at the $\mathrm{TiO}_{2}\left(\begin{array}{lll}1 & 1 & 0\end{array}\right)$ surface," Chemical Physics Letters, vol. 355, no. 5-6, pp. 417-423, 2002.

[19] B. J. Morgan and G. W. Watson, "A DFT + U description of oxygen vacancies at the $\mathrm{TiO}_{2}$ rutile $\left(\begin{array}{lll}1 & 1 & 0\end{array}\right)$ surface," Surface Science, vol. 601, no. 21, pp. 5034-5041, 2007. 


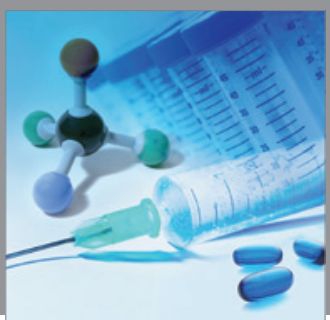

International Journal of

Medicinal Chemistry

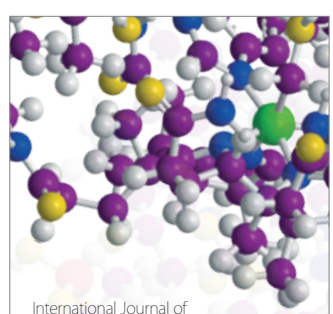

Carbohydrate Chemistry

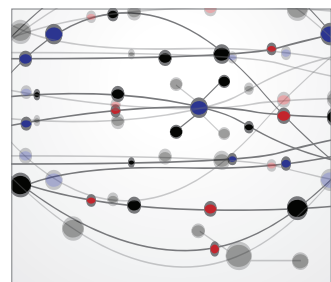

The Scientific World Journal
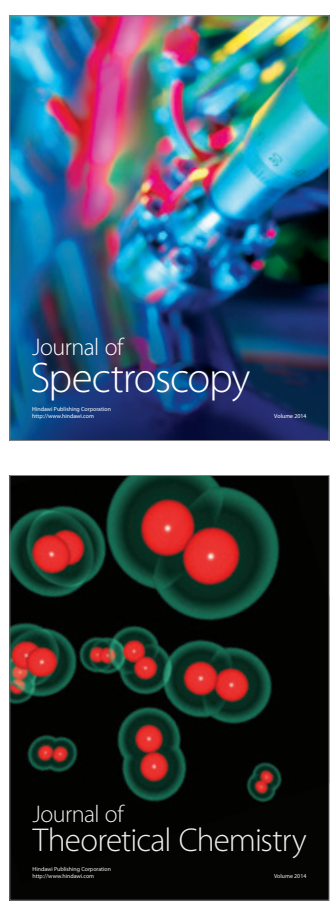
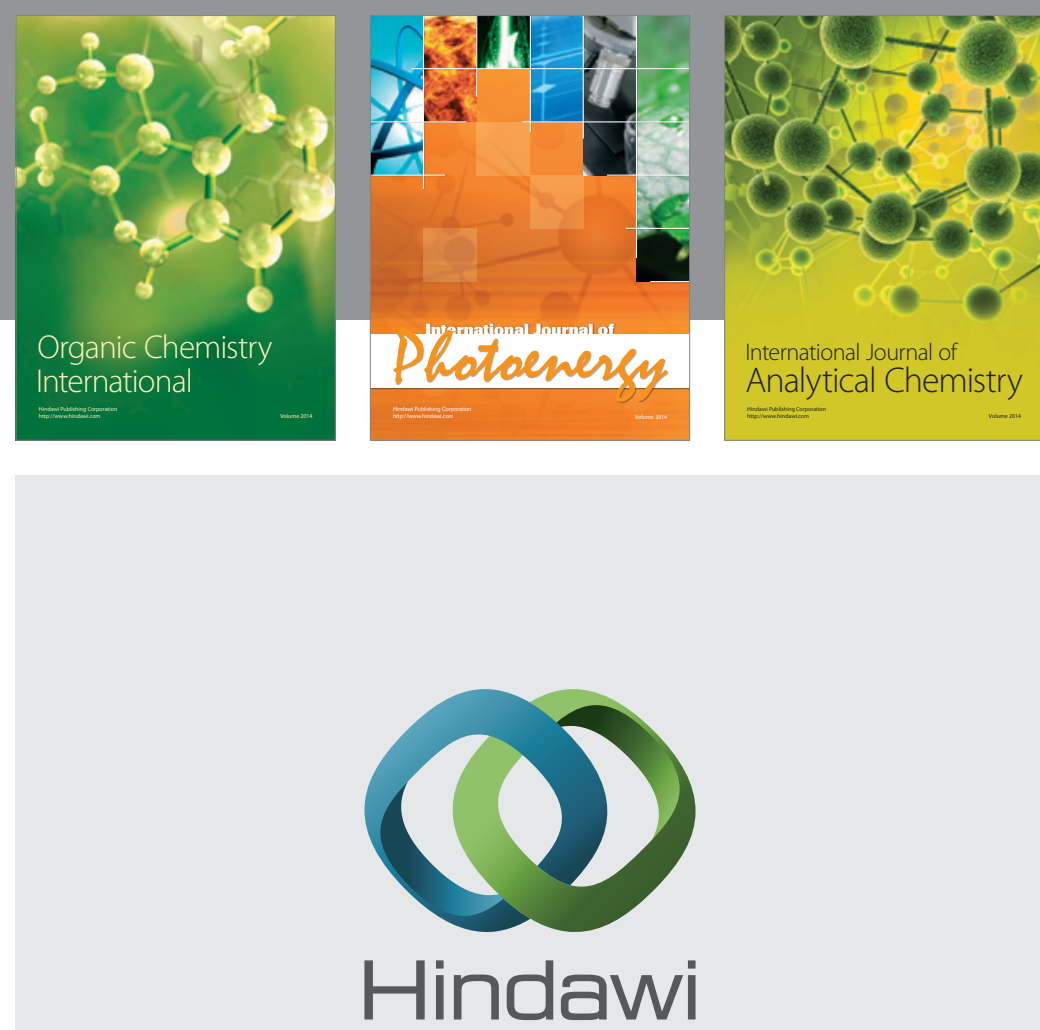

Submit your manuscripts at

http://www.hindawi.com
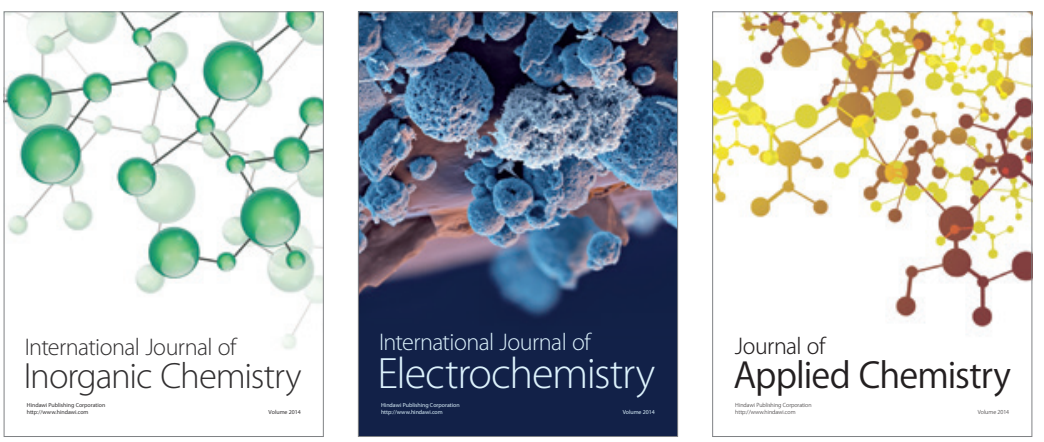

Journal of

Applied Chemistry
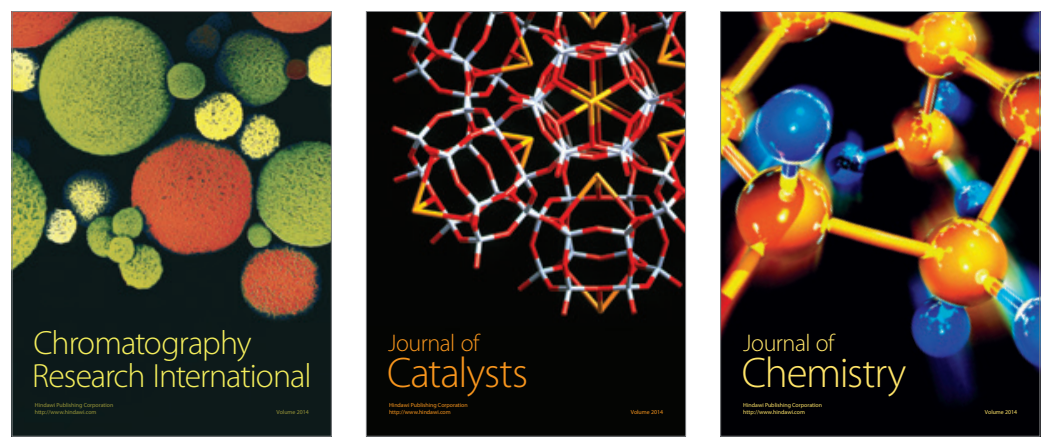
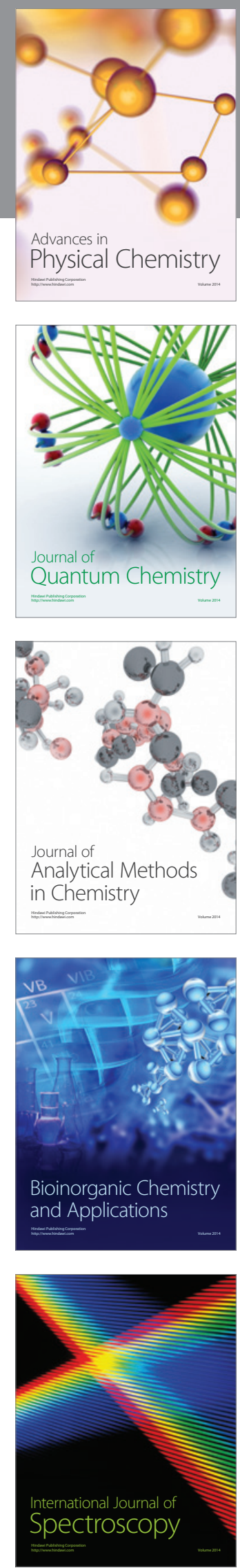